\title{
Percutaneous screw fixation for acute scaphoid fractures through K-wire-assisted reduction and maintenance:a randomized controlled trial
}

Jiangbo Bai

Hebei Medical University Third Affiliated Hospital

Lingde Kong

Hebei Medical University Third Affiliated Hospital

Siyu Tian

Hebei Medical University Third Affiliated Hospital

Kunlun Yu

Hebei Medical University Third Affiliated Hospital

Jian Lu

Hebei Medical University Third Affiliated Hospital

Chunjie Liu

Tangshan Gongren Hospital

Dehu Tian ( $\nabla$ tiandehu_1961@126.com )

Hebei Medical University Third Affiliated Hospital https://orcid.org/0000-0002-9409-9296

\section{Technical note}

Keywords: percutaneous fixation, scaphoid, acute fracture, displaced fracture

Posted Date: August 23rd, 2020

DOI: https://doi.org/10.21203/rs.3.rs-48050/v2

License: (c) (i) This work is licensed under a Creative Commons Attribution 4.0 International License. Read Full License 


\section{Abstract}

Background Most of the acute scaphoid fractures have been treated nonoperatively in a cast or by open surgery. Percutaneous screw fixation was introduced for acute scaphoid fractures through K-wire-assisted reduction and maintenance, and the effectiveness of the methods was evaluated. Methods From January 2015 to December 2018, a total of 20 patients were recruited to the study and 10 patients were randomly allocated to the open reduction and percutaneous groups respectively. Different groups received open reduction and internal fixation or percutaneous screw fixation. Function outcomes including the range of motion and grip strength were assessed according to the modified Mayo wrist scoring system. Results Final follow-up examination was performed on an average of 12 months after surgery. No immediate postoperative complication occurred. All patients achieved solid union as confirmed by CT scans. A significant increase in the functional recovery of the injured wrist in the percutaneous group than they were in the open reduction group. Conclusions Our novel percutaneous screw fixation method is beneficial to minimise injury to the blood supply of the scaphoid. Primary percutaneous screw fixation for acute scaphoid fractures is a superior method with reduced time to bony union, early return to daily activity or employment and predictably lessened complications of wrist stiffness, diminished grip strength, delayed union, non-union and osteonecrosis.

\section{Background}

The scaphoid is the most commonly fractured carpal bone in active adults and accounts for up to $80 \%$ of all carpal fractures[1,2]. Scaphoid fracture is mainly a consequence of fall with hypertension past $95^{\circ}$ and radial deviation of the wrist and axial loading from a direct blow[3,4]. The optimum treatment approach for acute scaphoid fractures is currently under discussion. Cast immobilisation is the main treatment for non-displaced scaphoid fractures, but approximately $20 \%$ of the cases fail to heal with conservative treatment[5]. Additionally, non-displaced scaphoid fractures usually require a period of cast immobilisation with time to union ranging from 8 weeks to 12 weeks. Long periods under this procedure may result in wrist stiffness, loss of grip strength, muscle atrophy and disuse osteopenia[6], thereby possibly affecting the function of wrist and hand. Screw fixation of scaphoid fractures has been recommended to minimise the period of cast immobilisation.

Displaced and unstable scaphoid fractures have a high risk of non-union and avascular necrosis[7], because these conditions damage the retrograde blood flow to the proximal fracture fragment. Operative treatment is mostly adopted for displaced and unstable scaphoid fractures. An open approach to the scaphoid can provide optimal visualisation of the fracture site for anatomical reduction and satisfactory screw location; however, this method has inherent disadvantages of ligament and capsular dissection and blood vessel damage[8]. In 1970, Streli R[9] adopted percutaneous screw fixation for scaphoid fractures to avoid this potential deficiency. This method could shorten the period of immobilisation and increase bone union rates. Separation of scaphoid fracture fragments commonly occurs during drilling and screw insertion. A gap is produced between fracture fragments and hinders fracture healing. 
The present study introduces a novel technique of percutaneous screw fixation for acute scaphoid fractures. One K-wire was used to maintain the reduction of scaphoid fractures during drilling, screw insertion and screw fixation for acute scaphoid fractures. To addres this issue, we compared the open reduction and internal fixation of acute scaphoid fractures with percutaneous screw fixation a randomized controlled study of patients after the injury.

\section{Methods}

This double-blind, randomized, single-center study was approved by the local ethics committee and in accordance with the declaration of Helsinki. From January 2015 to December 2018, patients were recruited according to the criteria described in Table 1 . The patients were referred to our institution, a university hand surgery section. Each patient was examined clinically and with plain radiography before being recruited into the study at the time of trauma. All included fractures were classified according to modified Herbert classification.

Patients were randomly assigned into 2 groups. Group allocation was performed according to a table of randomized numbers generated by a computer. A research assistant not associated with this study carried out the allocation process. After the patients were consented to the surgery, the operations were scheduled as soon as practically possible.An opaque envelope was attached to the patient's medical records which was opened before the operation.

\begin{tabular}{|c|c|}
\hline Inclusion criteria & Exclusion criteria \\
\hline Isolated scaphoid fracture & - Coexisting wrist fractures \\
\hline -Injury to surgery less than 10 days & -Patients with vascular injuried requiring revascularization \\
\hline $\begin{array}{l}\text { - The soft tissue allowed direct skin } \\
\text { closure }\end{array}$ & $\begin{array}{l}\text { Patients with preexisting symptoms due to a previous } \\
\text { known wrist injury }\end{array}$ \\
\hline -Radiographically mature skeleton & -Loss of skin substance requiring grafts or flaps \\
\hline $\begin{array}{l}\text {-Written informed consent to undergo the } \\
\text { surgical procedure }\end{array}$ & $\begin{array}{l}\text { Uncompensated diabetes, neoplasia, haemocoagulative } \\
\text { alterations, psychic disorders }\end{array}$ \\
\hline $\begin{array}{l}\text { Patients of either sex aged between } 18 \\
\text { and } 65 \text { years }\end{array}$ & -Transscaphoid perilunate dislocation \\
\hline
\end{tabular}

Table 1 List of inclusion and exclusion criteria of the study 
A total of 25 patients with scaphoid fracture were eligible for inclusion (Fig. 1). 5 patients withdrew and 20 patients received the allocated treatment. The 20 enrolled patients were randomly assigned to an open reduction group and a percutaneous group according to the different treatments applied. A team of 3 hand surgeons with at least five years of surgical experience performed all operations with the assistance of hand surgery residents.

Brachial plexus anaesthesia was provided. The patient was in a supine position with the operative arm abducted on the hand table. A pneumatic tourniquet was applied above the elbow, and an intravenous antibiotic was administered to prevent infection prior to the operation. 10 patients in the open reduction group were treated with open reduction and internal fixation. The fractures were exposed through a volar approach centered on the scaphoid. The scaphoid fracture after reduction was stabilized with a headless compression screw (Irene, Tianjin, China).

Volar percutaneous approach was applied to 10 patients in the percutaneous group. With the wrist placed in ulnar deviation, one K-wire was introduced perpendicularly through the styloid process of radius into the proximal pole of scaphoid under fluoroscopic guidance. The scaphoid fragment was reduced by dorsiflexing the wrist and translating the distal pole into an extended position. If the scaphoid fragment is not reduced successfully, then a Kwire is placed into the distal pole from the dorsal side of scaphoid to act as a joystick that reduces the fracture fragments (Fig.2 A,B). After the satisfactory reduction was achieved under fluoroscopic guidance, the starting point for the scaphoid screw was centred in the radial portion of the scaphoid tubercle. The wrist was dorsiflexed, supinated to bring the starting point of view. Scaphoid reduction was maintained while the wrist was dorsiflexed and supinated for placement of the central guidewire. A guide pin was placed down its central axis under fluoroscopic guidance and was driven across the fracture site into the proximal pole (Fig.2 C,D). Another anti-rotation wire was introduced parallel to the former guide pin. A hand reamer was then used to create a drill hole along the guide pin and insert a proper cannulated screw to the subchondral line at the proximal-most aspect of the scaphoid. Under the fixed proximal pole of scaphoid with radial styloid process, a headless compression screw (Irene, Tianjin, China) was inserted in standardised manner to avoid 
widening the fracture gap and achieve compression across the fracture site. After screw placement, screw position and satisfactory scaphoid reduction were confirmed by fluoroscopic imaging. All wires and anti-rotation pin were removed, and the tourniquet was released. The wound was irrigated and covered with a sterile dressing. Finally, the wrist was immobilised in the shirt arm volar splint, leaving the fingers and thumb free. The surgical process is shown in Fig. 3.

After surgery, the arm was elevated to promote venous drainage in the arm. Active digital range-of-motion exercises were started post-operatively. The patient returned for suture removal 2 weeks after surgery, and the splint was removed after 4 weeks. X-rays on AP, lateral, and dedicated scaphoid views were taken at 4, 8, and 12 weeks after surgery for fracture healing assessment. A CT scan was performed when adequate radiographic evidence union was obtained by plain radiographs.

Operation time, time to union, time to return to previous activity and complications were recorded. According to the modified Mayo wrist scoring system, function outcomes including pain, work status, range of motion (Rom) and grip strength were assessed and graded as excellent (91-100 scores), good (80-90 scores), fair (65-79 scores) and poor (less than 65 scores). Wrist range of motion (flexion, extension) and grip strength were measured by a Jamar dynamometer (Sammons Preston, Inc., Bollingbrook, IL, USA).

\section{Statistical methods}

The data were analysed using proprietary statistical software (SPSS 24.0; SPSS inc, United States). The Kolmogorov-Smirnov test was used to check whether the data conformed to a normal distribution. In case of normal distribution an independent student's t-test was performed to compare the mean active ranges of motion and grip strength between the groups. The modified Mayo wrist scoring was analysed with the chi-square test and Fisher exact test. P-values $<0.05$ were considered to indicate statistical significance. All tests were two-tailed.

\section{Results}


From January 2015 to December 2018, a total of 20 patients were recruited to the study and 10 patients were included in the 2 groups respectively. Demographic and clinical characteristics for each study group were comparable. There were no statistically significant differences between groups in either age $(p=0.39)$, sex $(p=1.0)$, occupation $(p=0.23)$, or fracture type $(p=0.37)$, etc. (Table 2$)$. Final follow-up examination was performed on an average of 12 months after surgery. No immediate postoperative complication occurred. All patients achieved solid union as confirmed by CT scans. Postoperative follow-up indicated that all patients in the percutaneous group exhibited excellent and good recovery according to the modified Mayo wrist scoring system, as shown in Fig. 4.

\begin{tabular}{|c|c|c|c|}
\hline Characteristics & $\begin{array}{c}\text { Patients Managed with } \\
\text { Percutaneous Screw Fixation }\end{array}$ & $\begin{array}{c}\text { Patients Managed with Open } \\
\text { Reduction and Internal Fixation }\end{array}$ & $\begin{array}{c}\mathrm{P} \\
\text { Value* }\end{array}$ \\
\hline$\overline{\text { Age (years) }}$ & $40.2 \pm 3.3$ & $35.5 \pm 4.1$ & 0.39 \\
\hline Sex (male : female) & $7: 3$ & $8: 2$ & 1.0 \\
\hline Smoking (yes : no) & $6: 4$ & $7: 3$ & 1.0 \\
\hline $\begin{array}{l}\text { Dominant : non-dominant } \\
\text { hands }\end{array}$ & $8: 2$ & $5: 5$ & 0.35 \\
\hline Hands $₫$ Right:Left $\square$ & 6:4 & 7:3 & 1.0 \\
\hline Occupation & & & 0.23 \\
\hline Office worker & 2 & 1 & \\
\hline Manual labourer & 6 & 9 & \\
\hline Sports player & 2 & 0 & \\
\hline Aetiology & & & 0.47 \\
\hline $\begin{array}{l}\text { Fall on an outstretched } \\
\text { hand }\end{array}$ & 8 & 10 & \\
\hline Traffic accident & 2 & 0 & \\
\hline $\begin{array}{l}\text { Duration from injury to } \\
\text { operation (ddys) }\end{array}$ & $3.8 \pm 0.29$ & $4.9 \pm 0.72$ & 0.17 \\
\hline $\begin{array}{l}\text { Fracture type(The } \\
\text { classification of Hebert) }\end{array}$ & & & 0.37 \\
\hline B1 & 4 & 7 & \\
\hline B2 & 6 & 3 & \\
\hline
\end{tabular}

Table 2. Patient Characteristics.

Continuous variables are expressed as mean with standard deviation(SD).

The means of the extension and flexion range of wrist joint in the open reduction group and percutaneous groups were $54.6^{\circ}, 61.1^{\circ}$ and $60.6^{\circ}, 66.1^{\circ}$ respectively. The means of the 
grip strength in the open reduction group and percutaneous groups were $35.4 \mathrm{kgand} 39.9$ $\mathrm{kg}$ respectively. The results of the two groups significantly differ between each other (Figure 5A-C).

The open reduction and percutaneous groups demonstrated 5, 10 excellent and good results, respectively. Statistical analysis showed a significant difference in the clinical grades of the outcomes between the groups according to the modified Mayo wrist scoring system(Figure 5D).

\section{Discussion}

The scaphoid comprises most of the carpel bones, and its fracture usually affects the mechanical integrity of wrist. The most important observation in this study was a significant increase in the functional recovery of the injured wrist in the percutaneous group than they were in the open reduction group.

Acute scaphoid fractures are usually classified as non-displaced and displaced fractures. Cast immobilisation is an adequate treatment for non-displaced scaphoid fracture[6,10]. Patients are being treated by below elbow scaphoid plaster cast with the thumb immobilised for a long time; however, the morbidity of cast immobilisation has recently attracted attention[11]. The conservative treatment of nondisplaced fractures does not allow early return to daily activity and work. When the forearm rotates in the plaster cast, all scaphoid fracture could displace from $1.0 \mathrm{~mm}$ to $4.0 \mathrm{~mm}[12]$. Thus, the non-displaced scaphoid fractures can be displaced in casting and high non-union rates. Acute displaced scaphoid fractures treated by casting have high rates of non-union and mal-union of up to $50 \%$ [13] Acute and other complications such as carpal instability, humpback deformity, joint stiffness, osteoarthritis, and atrophy of the upper extremity[14]. Operative treatment is offered for patients with acute scaphoid fractures to achieve both a high rate of fracture union and early return to daily activities[15].

The mechanical integrity of the wrist depends on the fascinating anatomy of the scaphoid. More than $70 \%$ of the scaphoid is covered by cartilage. Its blood supply is divided into extraosseous and intraosseous categories. Gelberman et al. described that nutrient arteries entering at the dorsal ridge supply the proximal $70 \%-80 \%$ of the scaphoid, and those entering at the tubercle supply the distal $20 \%-$ $30 \%[16]$. Morsy et al. reported that the scaphoid generally has two major networks of bony perfusion, one network entering volarly to supply the distal bone portion, and the other entering the dorsal ridge to supply the proximal portion. Approximately $83 \%$ of the scaphoid is supplied by the proximal vascular network, and $17 \%$ is supplied by the distal vascular network[17]. The main intraosseous vessels run in a retrograde flow to supply majority of the scaphoid. Scaphoid fractures proximal to the wrist can cut off the intraosseous blood supply to the proximal fragment and render the proximal fragment relatively avascular. As a result, the vulnerable blood supply to the proximal fragment greatly affects the scaphoid 
healing. Successful union of the scaphoid healing requires strains of less than $2 \%$. Any micromotion at the fracture site prevents callus formation and results in high non-union failure.

Open reduction and screw fixation of scaphoid fractures aim to promote callus formation and the associated economic productivity loss[18]. The open approach can provide optimal visualisation of the fracture site to achieve and maintain a satisfying reduction. In this approach, the dorsal capsular structure and volar radiocarpal ligament must be divided, which may damage the blood supply to the proximal fragment. In 1970, Streli [9] presented percutaneous compression screw for scaphoid fractures to avoid these shortcomings. The percutaneous volar or dorsal approach for scaphoid fractures has high union rate and low complication. The dorsal approach for proximal region fracture can provide convenience for percutaneous screw fixation; however, risk of damage occurs for the extensor of thumb and fingers, posterior interosseous and radiocarpal joint. Another disadvantage is that the screw destroys the proximal scaphoid cartilage and the native subchondral bone-cartilage interface, which is important in preventing arthritis[19]. Owing to the loosening or usage of long screws, proximal screw protrusion into the radiocarpal joint may occur post-operatively[20]. Adamany et al. described the palmar percutaneous screw fixation of scaphoid fractures for the first time. In contrast to the dorsal approach, the palmar approach is on the risk of tendon or nerve damage and screw protrusion to the radiocarpal joint[21]. Several authors have adopted the percutaneous technology for treating scaphoid fractures, but the scaphoid fracture might be displaced during drilling and screw insertion.

In this work, the proximal pole of scaphoid was transfixed with $1.5 \mathrm{~mm}$ wire placed percutaneously through the styloid process of radius to achieve and maintain the reduction of scaphoid fractures during operation. The fracture is reduced and stabilised in the wrist extension. Our technique has several advantages against operative measures. Firstly, anatomic reduction of scaphoid fracture can be achieved and maintained during operation. Secondly, this technique can avoid injury to scaphoid cartilage and relative ligaments and thus maintain the normal mechanical stability of the wrist. Thirdly, the blood supply to scaphoid fracture fragments is preserved by avoiding damage to extraosseous nutrient arteries. Finally, this technique can shorten the operation time and prevent patients from being exposed to X-ray radiation. Indications of this operation must be strictly confined to non-displaced and displaced acute scaphoid fractures; however, percutaneous screw fixation is not suitable for non-union and comminuted scaphoid fractures due to the following reasons. Firstly, the non-union of scaphoid needs debridement and cancellous bone graft for the bone defect. Secondly, the comminuted fractures need optimal visualisation of the fracture site to achieve anatomical reduction.

This study has several limitations. First is the small sample size; research with a large sample size is underway to provide evidence on the efficacy of this technique. Secondly, no case of small proximal pole less than $20 \%$ of scaphoid was included.

\section{Conclusions}


The novel percutaneous screw fixation can minimise injury to the blood supply of the scaphoid. Primary percutaneous screw fixation for acute scaphoid fractures is a superior method that reduces time to bony union, allows early return to daily activity or employment, and predictably lessens the complications of wrist stiffness, diminished grip strength, delayed union, non-union and osteonecrosis. Therefore, this percutaneous screw fixation must be considered as a useful and safe technique for acute scaphoid fractures.

\section{Declarations}

\section{Acknowledgements}

Not applicable.

\section{Funding}

This research received no specific grant from any funding agency in the public, commercial, or not-forprofit sectors.

\section{Availability of data and materials}

The datasets used and/or analyzed during the current study are available from the corresponding author on reasonable request.

\section{Authors' contributions}

The conception and design of the study were proposed by DHT, who also provided advice for revision of the manuscript. JBB collected, analyzed, and prepared the first draft of the paper. LDK, SYT and JL participated in the surgical procedures performed in the study. CJL and LDK contributed to the data collection and analysis. All authors read and approved the final manuscript.

\section{Ethics approval and consent to participate}

All protocols were approved by the Ethics Board of the Third Hospital of Hebei Medical University, and all participants provided informed consent. 


\section{Consent for publication}

Not applicable.

\section{Competing interests}

The authors declare that they have no competing interests.

\section{References}

[1] Hove LM (1999) Epidemiology of scaphoid fractures in Bergen, Norway. Scand J Plast Reconstr Surg Hand Surg 33:423-426.

[2] Arsalan-Werner A, Sauerbier M, Mehling IM (2016) Current concepts for the treatment of acute scaphoid fractures. Eur J Trauma Emerg Surg 42:3-10.

[3] Ibrahim T, Qureshi A, Sutton AJ, Dias JJ (2011) Surgical versus nonsurgical treatment of acute minimally displaced and undisplaced scaphoid waist fractures: pairwise and network meta-analyses of randomized controlled trials. J Hand Surg Am 36:1759-1768.

[4] Weber ER, Chao EY (1978) An experimental approach to the mechanism of scaphoid waist fractures. J Hand Surg Am 3:142-148.

[5] Mack GR, Wilckens JH, Mcpherson SA (1998) Subacute scaphoid fractures. A closer look at closed treatment. Am J Sports Med 26:56-58.

[6] Duppe H, Johnell O, Lundborg G, Karlsson M, Redlund-Johnell I (1994) Long-term results of fracture of the scaphoid. A follow-up study of more than thirty years. J BONE JOINT SURG AM 76:249-252.

[7] Grover R (1996) Clinical assessment of scaphoid injuries and the detection of fractures. J Hand Surg Br 21:341-343.

[8] Yu Y, Cui H, Yang X, Yu X, Bai Y (2018) A novel percutaneous achievement and maintenance of reduction and screw fixation for acute displaced scaphoid fractures: minimum two-year follow-up. INT ORTHOP 42:1911-1916.

[9] Streli R (1970) Percutaneous screwing of the navicular bone of the hand with a compression drill screw (a new method). Zentralbl Chir 95:1060-1078.

[10] Dias JJ, Brenkel IJ, Finlay DB (1989) Patterns of union in fractures of the waist of the scaphoid. J Bone Joint Surg Br 71:307-310. 
[11] Herbert TJ, Fisher WE (1984) Management of the fractured scaphoid using a new bone screw. J Bone Joint Surg Br 66:114-123.

[12] Merrell G, Slade J (2008) Technique for percutaneous fixation of displaced and nondisplaced acute scaphoid fractures and select nonunions. J Hand Surg Am 33:966-973.

[13] Weber ER (1980) Biomechanical implications of scaphoid waist fractures. Clin Orthop Relat Res149:83-89.

[14] Garcia RM, Ruch DS (2014) Management of scaphoid fractures in the athlete: open and percutaneous fixation. Sports Med Arthrosc Rev 22:22-28.

[15] Bond CD, Shin AY, Mcbride MT, Dao KD (2001) Percutaneous screw fixation or cast immobilization for nondisplaced scaphoid fractures. J BONE JOINT SURG AM 83:483-488.

[16] Gelberman RH, Menon J (1980) The vascularity of the scaphoid bone. J Hand Surg Am 5:508-513.

[17] Morsy M, Sabbagh MD, van Alphen NA, Laungani AT, Kadar A, Moran SL (2019) The Vascular Anatomy of the Scaphoid: New Discoveries Using Micro-Computed Tomography Imaging. J Hand Surg Am 44:928-938.

[18] Rettig ME, Kozin SH, Cooney WP (2001) Open reduction and internal fixation of acute displaced scaphoid waist fractures. J Hand Surg Am 26:271-276.

[19] Poole AR (2003) What type of cartilage repair are we attempting to attain? J BONE JOINT SURG AM 85-A Suppl 2:40-44.

[20] Megerle K, Keutgen X, Muller M, Germann G, Sauerbier M (2008) Treatment of scaphoid non-unions of the proximal third with conventional bone grafting and mini-Herbert screws: an analysis of clinical and radiological results. J Hand Surg Eur Vol 33:179-185.

[21] Adamany DC, Mikola EA, Fraser BJ (2008) Percutaneous fixation of the scaphoid through a dorsal approach: an anatomic study. J Hand Surg Am 33:327-331.

\section{Figures}




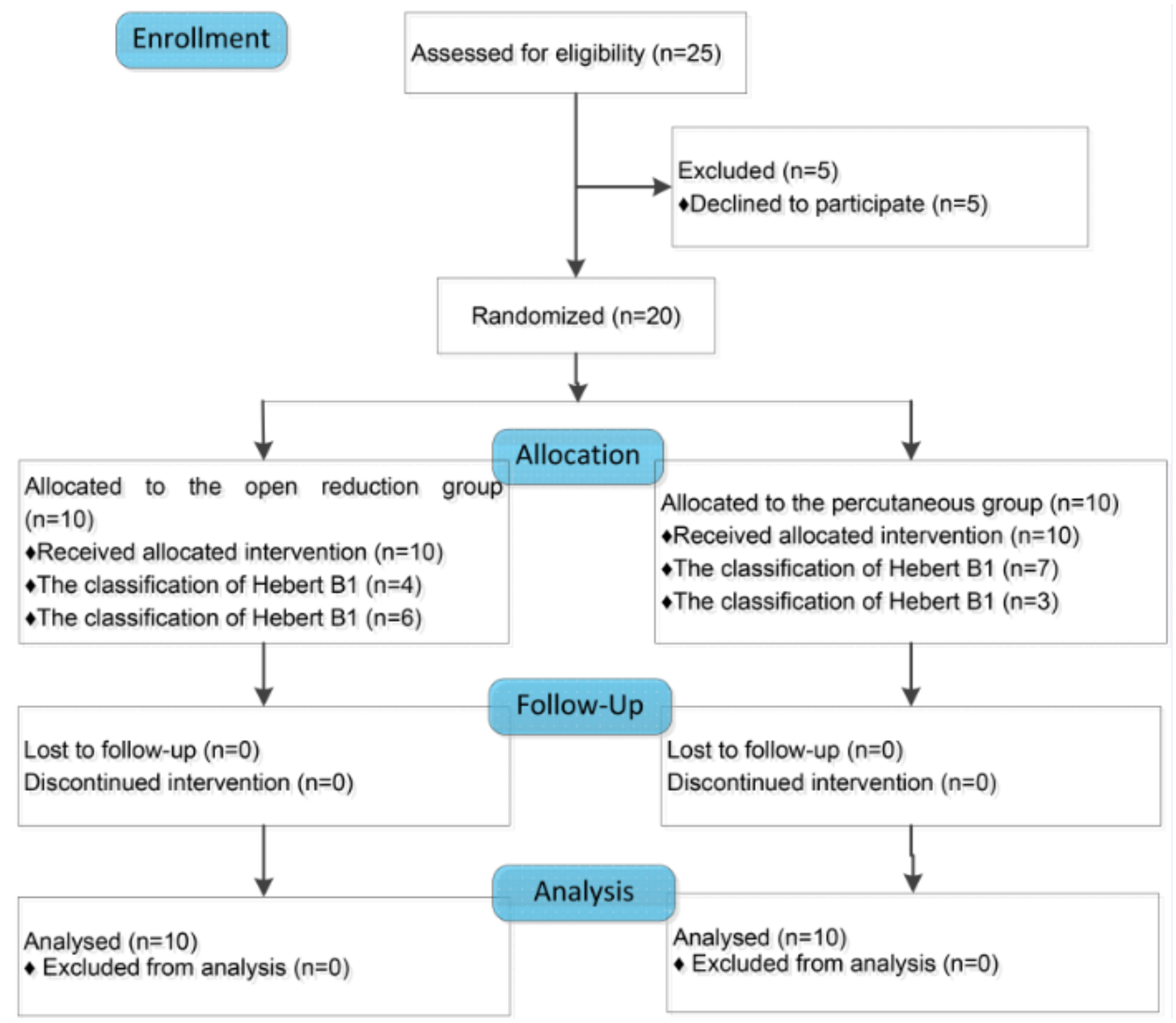

\section{Figure 1}

Diagram showing the flow of patients through each stage of the study. 

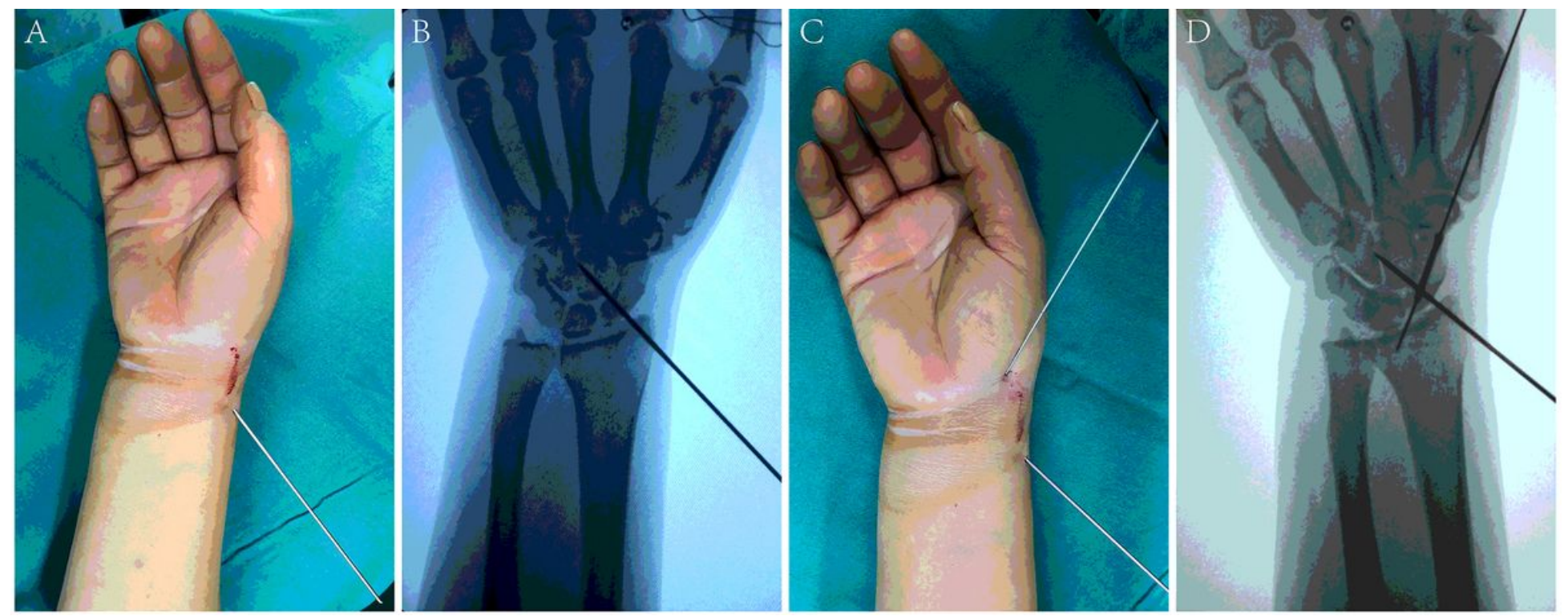

Figure 2

Final intraoperative fluoroscopy showing good fracture reduction assisted by the assisting K-wire $(A, B)$ and screw fixation across a scaphoid fracture(C,D).
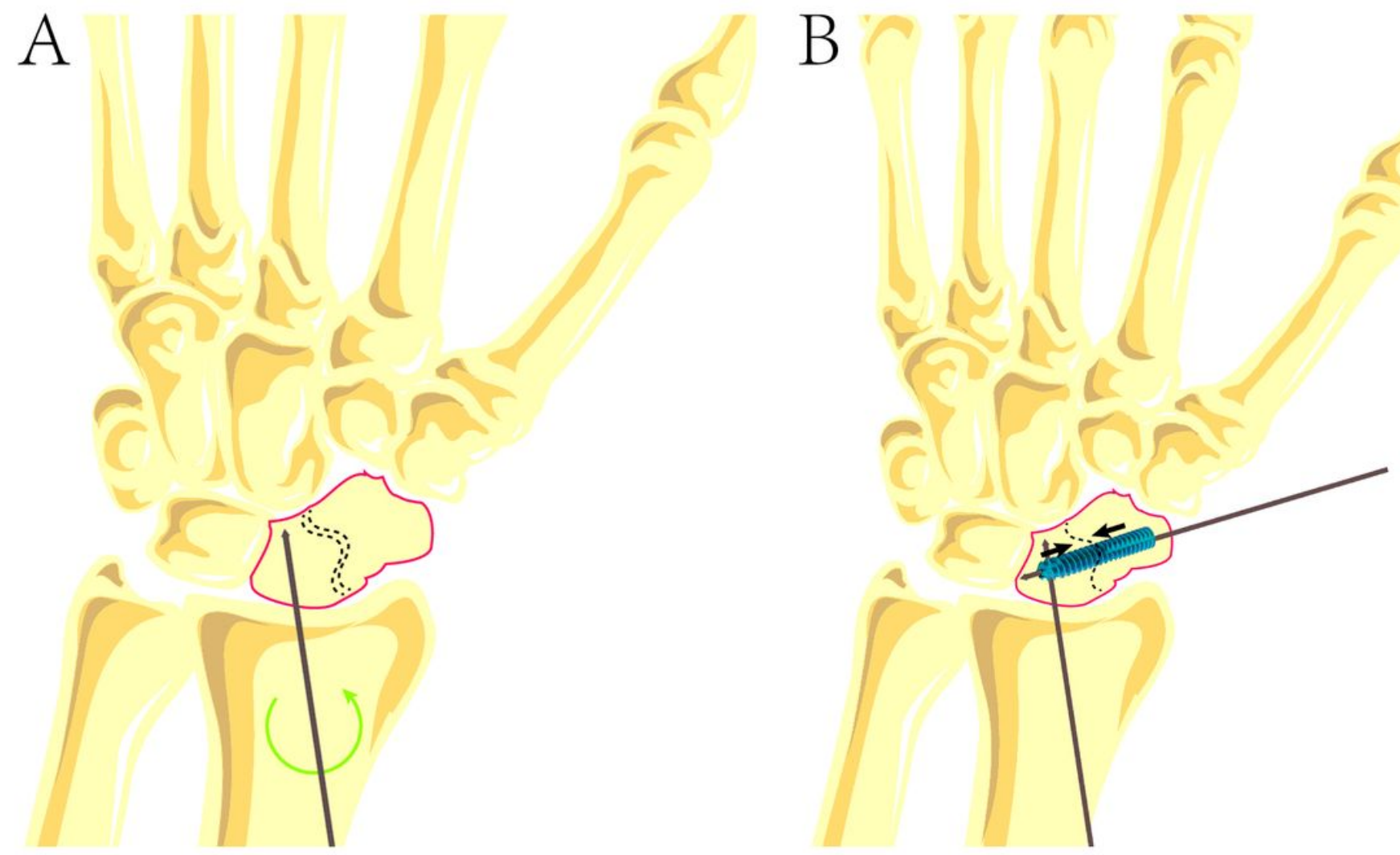

\section{Figure 3}

Schematic representation of scaphoid fracture in the waist. The dashed black line represents the fracture line. The green arrow represents reset direction of the assisting K-wire. A Reduction of scaphoid fracture assisted by the assisting K-wire B The image showing screw fixation across a scaphoid fracture. 


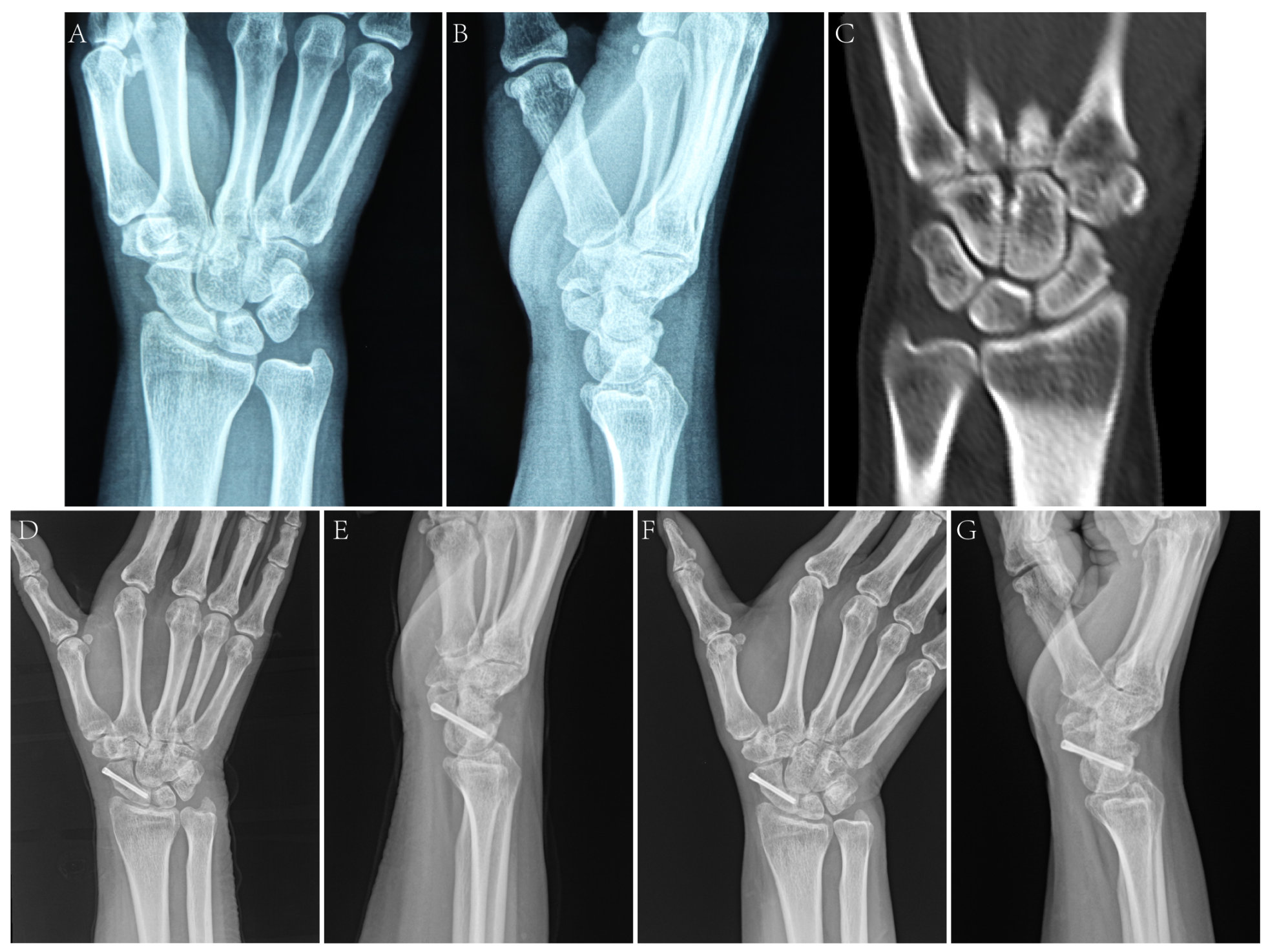

Figure 4

A 51-year-old woman with acute scaphoid fracture in the waist. A,B Preoperative anteroposterior and lateral radiographs; imaging on the day of injury. C Preoperative CT scans with sagittal views.Radiographs show fuzzy fracture line 1 month after the surgery(D,E). Radiographs show successful fracture healing 3 months after the surgery $(F, G)$. 
A Wrist range of motion (extension) $\left({ }^{\circ}\right)$

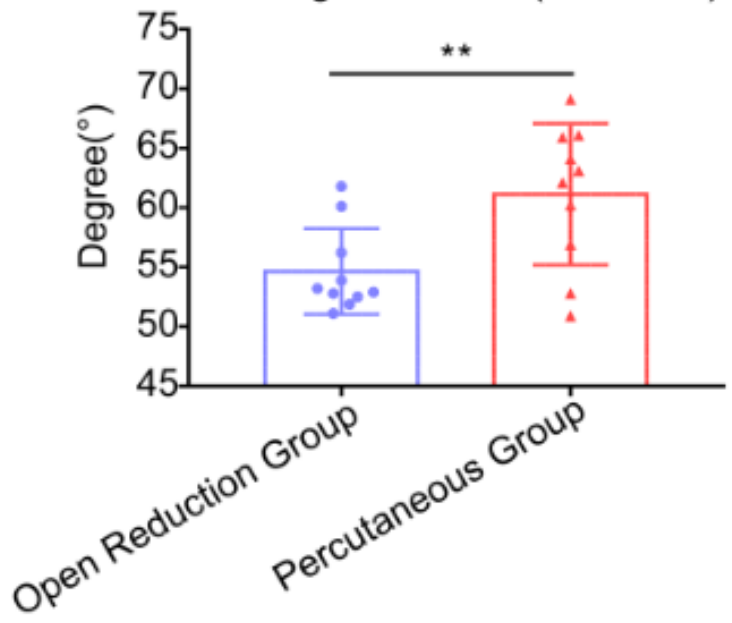

C

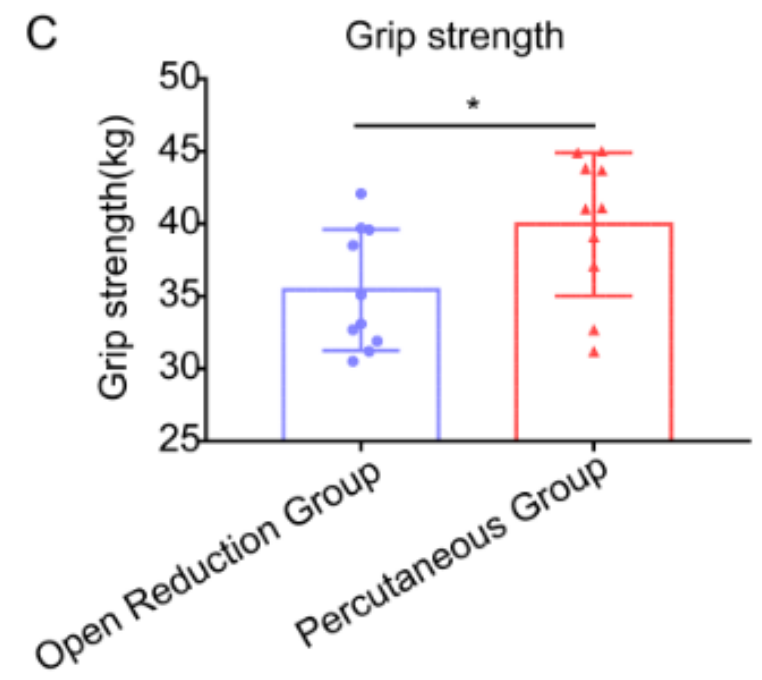

B

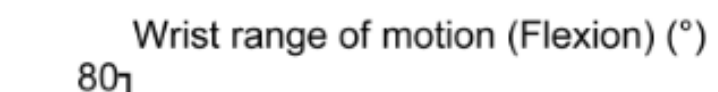

D

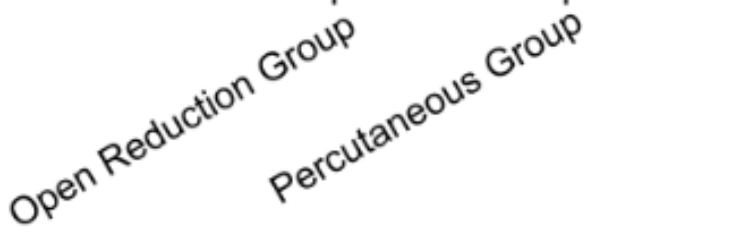

Mayo wrist scoring

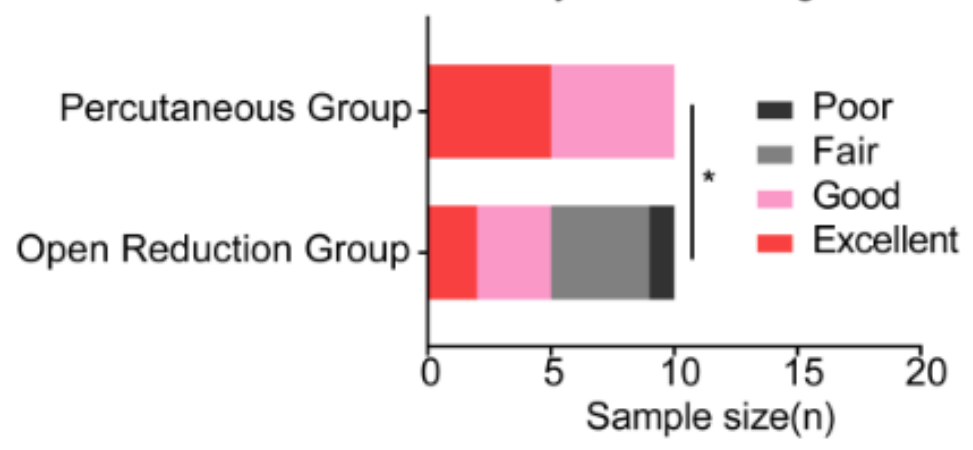

\section{Figure 5}

Mean range of motion $(A, B)$ and grip strength $(C)$ at follow-up assessments by visit. The percutaneous group was significantly different from the open reduction group. A significant difference in the clinical

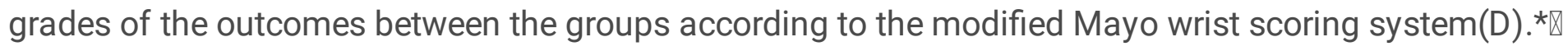
$p<0.05 ; * *: p<0.01$. 